\title{
Individualized chemotherapy for colorectal cancer based on the collagen gel droplet-embedded drug sensitivity test
}

\author{
TAKUMI OCHIAI ${ }^{1}$, KAZUHIKO NISHIMURA $^{1}$, TOMOO WATANABE ${ }^{1}$, MASAYUKI KITAJIMA $^{1}$, \\ AKINORI NAKATANI ${ }^{1}$, TAKASHI INOU ${ }^{1}$, MARIE WASHIO ${ }^{1}$, NAOKI SAKUYAMA ${ }^{1}$, \\ TSUYOSHI SATO $^{1}$, KENJI KISHINE ${ }^{1}$, TAKANORI OCHI ${ }^{1}$, SATOSHI OKUBO ${ }^{1}$, \\ SHUNJI FUTAGAWA ${ }^{1}$, SATOMI MASHIKO ${ }^{2}$ and ISAO NAGAOKA ${ }^{3}$ \\ Departments of ${ }^{1}$ Surgery and ${ }^{2}$ Pharmacy, Tobu Chiiki Hospital, Tokyo Metropolitan Health and \\ Medical Treatment Corporation; ${ }^{3}$ Department of Host Defense and Biochemical Research, \\ Juntendo University School of Medicine, Tokyo, Japan
}

Received March 20, 2012; Accepted June 26, 2012

DOI: $10.3892 / \mathrm{ol} .2012 .823$

\begin{abstract}
The leucovorin (FOL) and fluorouracil (5-FU) plus oxaliplatin (1-OHP; FOLFOX) or FOL and 5-FU plus irinotecan (SN-38; FOLFIRI) regimens with or without molecularly-targeted drugs are widely used as first-line chemotherapy in the treatment of advanced colorectal cancer (CRC). Whether FOLFOX or FOLFIRI is administered first is not significant, however, it is essential that full administration of the targeted dosages of all 3 drugs, 5-FU, 1-OHP and $\mathrm{SN}-38$, is achieved. However, this is not always possible and second-line chemotherapy must be abandoned in certain cases. Where possible, the most effective regimen should be selected as the first line of treatment. The aim of this study was to determine whether first-line chemotherapy may be individualized using the collagen gel droplet-embedded drug sensitivity test (CD-DST). Specimens of primary tumors were obtained from 43 CRC patients who had received no preoperative chemotherapy. Informed consent to measure drug sensitivity was obtained from all patients. The CD-DST allows evaluation of drug sensitivity using isolated, 3-dimensionally cultured tumor cells in a small collagen gel droplet. The CD-DST was performed and the growth inhibition rate (IR) was obtained under incubation conditions (5-FU with 1-OHP at 6.0 and $3.0 \mu \mathrm{g} / \mathrm{ml}$, or 5-FU with SN-38 at 6.0 and $0.2 \mu \mathrm{g} / \mathrm{ml}$, respectively, for $24 \mathrm{~h}$ ). The cumulative distributions of the growth IRs under each condition were evaluated based on the evidence that the clinical response rates to FOLFOX and FOLFIRI were almost the same. Individualization of
\end{abstract}

Correspondence to: Dr Takumi Ochiai, Department of Surgery, Tobu Chiiki Hospital, Tokyo Metropolitan Health and Medical Treatment Corporation, 5-14-1 Kameari, Katsushika, Tokyo 125-8512, Japan

E-mail: takumi-o@ma.kitanet.ne.jp

Key words: colon cancer, individualized chemotherapy, collagen gel droplet-embedded drug sensitivity test, leucovorin and fluorouracil plus oxaliplatin, leucovorin and fluorouracil plus irinotecan first-line treatment was possible in all patients, with FOLFOX and FOLFIRI showing higher efficacy in 26 and 15 patients, respectively, and equal efficacy in 2 cases. This method has the potential to facilitate the establishment of individualized first-line chemotherapy for CRC and improve the prognosis in such patients.

\section{Introduction}

The leucovorin (FOL) and fluorouracil (5-FU) plus oxaliplatin (1-OHP; FOLFOX) or leucovorin and 5-FU plus irinotecan (SN-38; FOLFIRI) regimens with or without molecularlytargeted drugs are widely used as first-line chemotherapy in the treatment of advanced colorectal cancer (CRC) (1-12). Whether FOLFOX or FOLFIRI is administered first is not significant, however, it is crucial that full administration of the targeted dosages of all 3 drugs, 5-FU, 1-OHP and SN-38, is achieved. However, this is not always possible and second-line chemotherapy must be abandoned in certain cases due to disease progression, adverse effects or high medical costs $(13,14)$.

Where possible, the most effective regimen should be selected as the first line of treatment. A previous study using the collagen gel droplet embedded culture-drug sensitivity test (CD-DST) reported that FOLFIRI should be selected as the first line of chemotherapy in the treatment of poor responders to 5-FU (15).

The aim of the current study was to determine whether first-line chemotherapy may be individualized using the CD-DST.

\section{Patients and methods}

Patients. Specimens of primary tumors were obtained between March 2008 and September 2011 from 43 CRC patients who had received no preoperative chemotherapy. Informed consent for measuring drug sensitivity was obtained from all patients. The study was approved by the ethics committee at Juntendo University School of Medicine, Tokyo, Japan. 
Table I. Patient characteristics.

\begin{tabular}{lc}
\hline Characteristics & $\mathrm{n}$ \\
\hline No. of patients & 43 \\
Age (years), mean (range) & $64.3(42-78)$ \\
Gender (male/female) & $24 / 19$ \\
Histological type & \\
Well-differentiated carcinoma & 7 \\
Moderately differentiated carcinoma & 29 \\
Poorly differentiated carcinoma & 2 \\
Mucinous carcinoma & 5 \\
Colon/rectum & $35 / 8$ \\
Dukes' stage (A/B/C/D) & $2 / 13 / 19 / 9$ \\
\hline
\end{tabular}

Methods. Tumor tissue was excised from primary surgical specimens and subjected to the CD-DST. The CD-DST allows for the evaluation of drug sensitivity using isolated, 3-dimensionally cultured tumor cells in a small collagen gel droplet and was used to evaluate the sensitivity of the tumors to 5-FU, performed as described by Kobayashi et al $(16,17)$. Each specimen was washed 5 times with $50 \mathrm{ml}$ saline, followed by further washing 5 times with $50 \mathrm{ml}$ antibiotic fluid containing $1.0 \mathrm{mg} / \mathrm{ml}$ piperacillin and $0.5 \mathrm{mg} / \mathrm{ml} \mathrm{kanamycin}$. The transport bottle contained $1.0 \mathrm{mg} / \mathrm{ml}$ piperacillin, $0.5 \mathrm{mg} / \mathrm{ml}$ kanamycin and $2.5 \mu \mathrm{g} / \mathrm{ml}$ amphotericin B. Tissue (1 g) was treated for $2 \mathrm{~h}$ with a dispersion enzyme cocktail containing $1.0 \%$ collagenase. Dispersed cell suspensions were inoculated into pre-culture media on collagen-coated flasks overnight, after which viable tumor cells were recovered by $0.05 \%$ collagenase treatment. Recovered cells were embedded in $30-\mu 1$ collagen gel droplets.

The embedded cells were cultivated in culture media containing 5-FU and 1-OHP at 6.0 and $3.0 \mu \mathrm{g} / \mathrm{ml}$, or 5-FU and SN-38 at 6.0 and $0.2 \mu \mathrm{g} / \mathrm{ml}$, respectively, for $24 \mathrm{~h}$. Following the removal of the anticancer agent-containing media, the cells were further cultured for 7 days in serum-free culture media to prevent the growth of fibroblasts. Viable cells were stained with neutral red solution and counted using the imaging colorimetric quantification method. The surviving cell number ratio between the drug-treated and control group, which received no drug treatment, was calculated. A growth rate $<0.8$ was considered indicative of successful culture.

The frequency distributions of the growth inhibition rates (IRs) under the two conditions were evaluated based on the evidence that the clinical response rates to FOLFOX and FOLFIRI were almost the same.

Statistical analysis. Histograms were analyzed with the D'Agostino-Pearson omnibus normality test using GraphPad Prism (GraphPad Software, La Jolla, CA, USA). P $<0.05$ was considered to indicate statistically significant differences.

\section{Results}

Individualization of first-line chemotherapy is possible in patients with advanced CRC. Patient characteristics are shown
Table II. Individual growth inhibition rates.

\begin{tabular}{|c|c|c|}
\hline $\begin{array}{l}\text { Patient } \\
\text { no. }\end{array}$ & $\begin{array}{c}\text { 5-FU/1-OHP } \\
6.0 / 3.0 \mu \mathrm{g} / \mathrm{ml} \text { for } 24 \mathrm{~h}\end{array}$ & $\begin{array}{c}\text { 5-FU/SN-38 } \\
6.0 / 0.2 \mu \mathrm{g} / \mathrm{ml} \text { for } 24 \mathrm{~h}\end{array}$ \\
\hline 1 & 80.1 & 82.9 \\
\hline 2 & 71.3 & 79.2 \\
\hline 3 & 81.2 & 83.4 \\
\hline 4 & 60.0 & 68.7 \\
\hline 5 & 29.9 & 66.5 \\
\hline 6 & 69.7 & 89.6 \\
\hline 7 & 58.7 & 63.2 \\
\hline 8 & 73.0 & 85.2 \\
\hline 9 & 63.2 & 75.9 \\
\hline 10 & 77.9 & 85.5 \\
\hline 11 & 76.3 & 85.6 \\
\hline 12 & 53.6 & 62.6 \\
\hline 13 & 41.9 & 60.7 \\
\hline 14 & 81.3 & 80.9 \\
\hline 15 & 42.3 & 70.2 \\
\hline 16 & 84.8 & 86.8 \\
\hline 17 & 75.9 & 83.9 \\
\hline 18 & 59.2 & 76.4 \\
\hline 19 & 69.9 & 85.5 \\
\hline 20 & 57.0 & 49.7 \\
\hline 21 & 79.2 & 83.0 \\
\hline 22 & 86.1 & 89.1 \\
\hline 23 & 67.3 & 74.4 \\
\hline 24 & 81.3 & 85.2 \\
\hline 25 & 60.4 & 71.9 \\
\hline 26 & 93.4 & 98.6 \\
\hline 27 & 62.6 & 84.9 \\
\hline 28 & 58.5 & 54.8 \\
\hline 29 & 81.2 & 84.0 \\
\hline 30 & 66.5 & 73.3 \\
\hline 31 & 81.3 & 78.1 \\
\hline 32 & 59.9 & 74.1 \\
\hline 33 & 53.3 & 65.0 \\
\hline 34 & 49.3 & 48.3 \\
\hline 35 & 44.7 & 49.3 \\
\hline 36 & 68.8 & 72.1 \\
\hline 37 & 59.7 & 69.4 \\
\hline 38 & 50.8 & 59.3 \\
\hline 39 & 51.6 & 56.5 \\
\hline 40 & 57.9 & 70.2 \\
\hline 41 & 58.5 & 63.7 \\
\hline 42 & 62.4 & 72.5 \\
\hline 43 & 82.9 & 84.3 \\
\hline
\end{tabular}

5-FU, fluorouracil; 1-OHP, oxaliplatin; SN-38, irinotecan.

in Table I. The individual growth IRs under each of the two conditions are shown in Table II. With 5-FU and 1-OHP at 6.0 and $3.0 \mu \mathrm{g} / \mathrm{ml}$, respectively, the median, mean, standard deviation and standard error of the mean were 63.20, 65.69, 


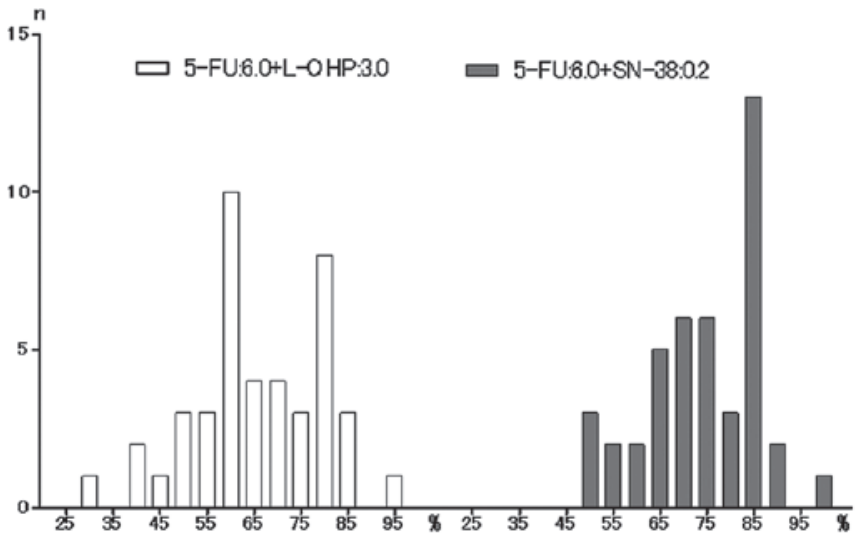

Histograms of individual growth inhibition rates

Figure 1. Histograms of individual growth inhibition rate (\%) in culture media containing 5-FU and 1-OHP at 6.0 and $3.0 \mu \mathrm{g} / \mathrm{ml}$, respectively, for $24 \mathrm{~h}$ or 5 -FU and SN-38 at 6.0 and $0.2 \mu \mathrm{g} / \mathrm{ml}$, respectively, for $24 \mathrm{~h} .5-\mathrm{FU}$, fluorouracil; 1-OHP, oxaliplatin; SN-38, irinotecan.

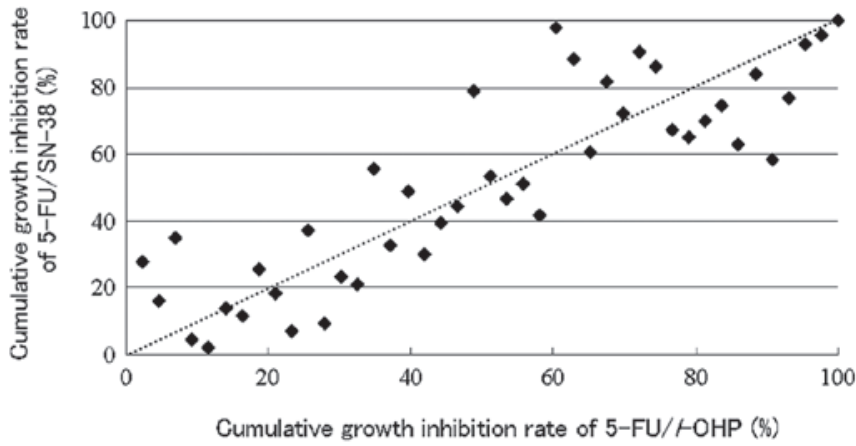

Figure 2. Correlation between cumulative distribution of individual growth inhibition rate between each condition. Dotted line indicates equivalence in efficacy. FOLFIRI was superior to FOLFOX in upper left half. FOLFOX was superior to FOLFIRI in lower right half. 5-FU, fluorouracil; 1-OHP, oxaliplatin;FOL, leucovorin; FOLFOX, FOL, 5-FU and 1-OHP; SN-38, irinotecan; FOLFIRI, FOL, 5-FU and SN-38.

14.02 and 2.138, respectively. With 5-FU and SN-38 at 6.0 and $0.2 \mu \mathrm{g} / \mathrm{ml}$, respectively, the median, mean, standard deviation and standard error of the mean were 74.40, 74.06, 12.12 and 1.848 , respectively. Histograms of the individual growth IRs (\%) under each of the two conditions are shown in Fig. 1. The histograms passed the normality test $(\alpha=0.05 ; 5$-FU and 1-OHP, $\mathrm{P}=0.7265$; 5-FU and $\mathrm{SN}-38, \mathrm{P}=0.3756$ ).

The cumulative distribution of the individual growth IRs between the two conditions is shown in Fig. 2. There are individual differences of the efficacies between the two regimens.

Individualization of first-line chemotherapy was possible in all patients, with 5-FU plus 1-OHP and 5-FU plus SN-38 showing higher efficacy in 26 and 15 patients, respectively, and equal efficacy in 2 cases (Fig. 2).

\section{Discussion}

The addition of molecularly-targeted anticancer agents enhances the effect of FOLFOX/FOLFIRI therapies. Moreover, individualized chemotherapy with molecularly-targeted anticancer agents may be implemented based on the genetic characteristics of the individual patient (6-12,18-20). Several studies have investigated individualization in 5-FU-based chemotherapy $(21,22)$. The three enzymes that have been identified as the most significant in the metabolism of 5-FU are orotate phosphoribosyl transferase (OPRT), thymidylate synthase (TS) and dihydropyrimidine dehydrogenase (DPD). The most significant phosphorylation enzyme of 5-FU is OPRT, while the degradation enzyme is DPD and the main enzyme of DNA synthesis is TS. In general, high expression of TS correlates with poor efficacy of 5-FU, while low expression correlates with good efficacy. The activity of these enzymes has been reported to be extremely useful in individualization of 5-FU chemotherapy in CRC (23). Moreover, the individual $50 \%$ inhibitory area under the concentration curve of 5-FU using the CD-DST has been reported to be useful in determining individualized chemotherapy in CRC patients $(24,25)$. Previous studies have investigated the correlation between the efficacy of irinotecan and topoisomerase-1, or that between the toxicity of irinotecan and uridine diphosphate glucuronosyltransferases $1 \mathrm{~A} 1(21,22,26-29)$. It has been reported that there is a correlation between the efficacy of oxaliplatin and the excision cross-complementing gene $(21,22,26)$. However, individualization in 5-FU-based chemotherapy remains to be implemented clinically.

Grothey et al reported that while it was not significant whether FOLFOX or FOLFIRI was administered first, it was crucial that full administration of the targeted dosages of all 3 drugs, 5-FU, oxaliplatin and irinotecan, be achieved. However, certain randomized controlled trials noted that, even with the best prognosis, full administration of all 3 drugs was not possible in approximately one-quarter of patients $(13,14)$. It has also been reported that second-line chemotherapy could not be carried out in approximately one-third of patients $(1,2,4,30,31)$. First-line chemotherapy is usually administered over a long period of time (5). Therefore, a more effective regimen should be selected. That is, if prognosis is to be improved, then individualization of first-line chemotherapy is indispensable.

It has been reported that the clinical response rates of FOLFOX and FOLFIRI are almost the same, at approximately $50 \%$ (5) and thus the efficacies of FOLFOX and FOLFIRI are considered to be almost equivalent $(1,2,4,5,30)$. However, it remains to be clarified whether this holds true if considered on a case-to-case basis. In this study, when the clinical response rates of FOLFOX and FOLFIRI were 50\%, responders were identified using the median based on the histograms of the individual growth IRs. The results also demonstrated that the efficacies of FOLFOX and FOLFIRI were not exactly equivalent in all the individuals in this study. Therefore, the more effective regimen for each individual was identified based on the cumulative distributions of the individual growth IRs between FOLFOX and FOLFIRI. FOLFOX was recommended as first-line chemotherapy in 26 patients, while FOLFIRI was recommended as first-line chemotherapy in 15 patients. Thus, individualization of first-line chemotherapy was possible in all patients.

The results from the present study suggest that this method has the potential to facilitate the establishment of individualized first-line chemotherapy for CRC and is likely to improve 
the prognosis in such patients. This method requires further prospective randomized study.

\section{Acknowledgements}

The authors would like to thank Associate Professor Jeremy Williams, Tokyo Dental College, for his assistance with the English of the manuscript.

\section{References}

1. de Gramont A, Figer A, Seymour M, et al: Leucovorin and fluorouracil with or without oxaliplatin as first-line treatment in advanced colorectal cancer. J Clin Oncol 18: 2938-2947, 2000.

2. Goldberg RM, Sargent DJ, Morton RF, et al: A randomized controlled trial of fluorouracil plus leucovorin, irinotecan, and oxaliplatin combinations in patients with previously untreated metastatic colorectal cancer. J Clin Oncol 22: 23-30, 2004.

3. Rothenberg ML, Oza AM, Bigelow RH, et al: Superiority of oxaliplatin and fluorouracil-leucovorin compared with either therapy alone in patients with progressive colorectal cancer after irinotecan and fluorouracil-leucovorin: interim results of a phase III trial. J Clin Oncol 21: 2059-2069, 2003

4. Douillard JY, Cunningham D, Roth AD, et al: Irinotecan combined with fluorouracil compared with fluorouracil alone as first-line treatment for metastatic colorectal cancer: a multicentre randomized trial. Lancet 355: 1041-1047, 2000.

5. Tournigand C, André T, Achille E, et al: FOLFIRI followed by FOLFOX6 or the reverse sequence in advanced colorectal cancer: a randomized GERCOR study. J Clin Oncol 22: 229-237, 2004.

6. Hochster HS, Hart LL, Ramanathan RK, et al: Safety and efficacy of oxaliplatin and fluoropyrimidine regimens with or without bevacizumab as first-line treatment of metastatic colorectal cancer: results of the TREE Study. J Clin Oncol 21: 3523-3529, 2008.

7. Saltz LB , Clarke S, Díaz-Rubio E, et al: Bevacizumab in combination with oxaliplatin-based chemotherapy as first-line therapy in metastatic colorectal cancer: a randomized phase III study. J Clin Oncol 26: 2013-2019, 2008.

8. Giantonio BJ, Catalano PJ, Meropol NJ, et al: Bevacizumab in combination with oxaliplatin, fluorouracil and leucovorin (FOLFOX4) for previously treated metastatic colorectal cancer: results from the eastern cooperative oncology group study E3200. J Clin Oncol 25: 1539-1544, 2007.

9. Bokemeyer C, Bondarenko I, Makhson A, et al: Fluorouracil, leucovorin and oxaliplatin with and without cetuximab in the first-line treatment of metastatic colorectal cancer. J Clin Onco 27: 663-671, 2009

10. Maughan TS, Adams R, Smith CG, et al: Identification of potentially responsive subsets when cetuximab is added to oxaliplatin-fluoropyrimidine chemotherapy (CT) in first-line advanced colorectal cancer (aCRC). J Clin Oncol 28 (Suppl 5): abs. 3502, 2010.

11. Fuchs CS, Marshall J, Mitchell E, et al: Randomized, controlled trial of irinotecan plus infusional, bolus, or oral fluoropyrimidines in first-line treatment of metastatic colorectal cancer: results from the BICC-C Study. J Clin Oncol 25: 4779-4786, 2007.

12. Van Cutsem E, Köhne CH, Hitre E, et al: Cetuximab and chemotherapy as initial treatment for metastatic colorectal cancer. $\mathrm{N}$ Eng J Med 360: 1408-1417, 2009.

13. Grothey A, Sargent D, Goldberg RM, et al: Survival of patients with advanced colorectal cancer improves with the availability of fluorouracil-leucovorin, irinotecan and oxaliplatin in the course of treatment. J Clin Oncol 22: 1209-1214, 2004.

14. Grothey A and Sargent D: Overall survival of patients with advanced colorectal cancer correlates with availability of fluorouracil, irinotecan and oxaliplatin regardless of whether doublet or single-agent therapy is used first line. J Clin Oncol 23: 9441-9442, 2005.
15. Ochiai T, Nishimura K, Watanabe T, et al: Leucovorin and fluorouracil plus oxaliplatin or leucovorin and fluorouracil plus irinotecan as individualized first-line therapy based on a drug sensitivity test. Exp Ther Med 1: 325-329, 2010.

16. Kobayashi H, Tanisaka K, Doi O, et al: An in vitro chemosensitivity test for solid tumors using collagen gel droplet embedded cultures. Int J Oncol 11: 449-455, 1997.

17. Kobayashi H,Higashiyama M,Minamigawa K, et al: Examination of in vitro chemosensitivity test using collagen droplet culture method with colorimetric endpoint quantification. Jpn J Cancer Res 92: 203-210, 2001.

18. Van Cutsem E, Köhne CH, Láng I, et al: Cetuximab plus irinotecan, fluorouracil and leucovorin as first-line treatment for metastatic colorectal cancer: updated analysis of overall survival according to tumor KRAS and BRAF mutation status. J Clin Oncol 29: 2011-2019, 2011

19. Bokemeyer C, Bondarenko I, Hartmann JT, et al: Efficacy according to biomarker status of cetuximab plus FOLFOX-4 as first-line treatment for metastatic colorectal cancer: the OPUS study. Ann Oncol 22: 1535-1546, 2011.

20. Douillard JY, Siena S, Cassidy J, et al: Randomized, phase III trial of panitumumab with infusional fluorouracil, leucovorin, and oxaliplatin (FOLFOX4) versus FOLFOX4 alone as first-line treatment in patients with previously untreated metastatic colorectal cancer: the PRIME study. J Clin Oncol 28: 4697-4705, 2010.

21. Koopman M, Venderbosch S, van Tinteren H, et al: Predictive and prognostic markers for the outcome of chemotherapy in advanced colorectal cancer, a retrospective analysis of the phase III randomized CAIRO study. Eur J Cancer 45: 1999-2006, 2009.

22. Koopman M, Venderbosch S, Nagtegaal ID, van Krieken JH and Punt CJ: A review on the use of molecular markers of cytotoxic therapy for colorectal cancer, what have we learned? Eur J Cancer 45: 1935-1949, 2009.

23. Ochiai T, Nishimura K, Noguchi H, et al: Prognostic impact of orotate phosphoribosyl transferase among 5-fluorouracil metabolic enzymes in resectable colorectal cancers treated by oral 5-fluorouracil-based adjuvant chemotherapy. Int J Cancer 118: 3084-3088, 2006.

24. Ochiai T, Nishimura K, Watanabe T, et al: Evaluation of the individual $50 \%$ inhibitory area under the concentration curve of 5-fluorouracil based on the collagen gel droplet embedded culture drug sensitivity test in colorectal cancer. Mol Med Rep 2: 405-409, 2009.

25. Ochiai T, Nishimura K, Watanabe T, et al: Personalized adjuvant chemotherapy for colorectal cancer (CRC) based on an individual $50 \%$ inhibitory area under the concentration curve (AUC) using collagen gel droplet embedded culture-drug sensitivity test (CD-DST). J Clin Oncol 28 (Suppl 15): abs. 3582, 2010.

26. Braun MS, Richman SD, Quirke P, et al: Predictive biomarkers of chemotherapy efficacy in colorectal cancer: results from the UK MRC FOCUS trial. J Clin Oncol 26: 2690-2698, 2008.

27. Minami H, Sai K, Saeki M, et al: Irinotecan pharmacokinetics/pharmacodynamics and UGT1A genetic polymorphisms in Japanese: roles of UGT1A $1 * 6$ and $* 28$. Pharmacogenet Genomics 17: 497-504, 2007.

28. Ando Y, Saka H, Ando M, et al: Polymorphisms of UDP-glucuronosyltransferase gene and irinotecan toxicity: a pharmacogenetic analysis. Cancer Res 60: 6921-6926, 2000.

29. Sai K, Sawada J and Minami H: Irinotecan pharmacogenetics in Japanese cancer patients: roles of UGT1A1 $* 6$ and $* 28$. Yakugaku Zasshi 128: 575-584, 2008 (In Japanese).

30. Seymour MT, for the UK NCRI colorectal clinical studies group: Fluorouracil, oxaliplatin and CPT-11 (irinotecan), use and sequencing (MRC FOCUS): a 2135-patient randomized trial in advanced colorectal cancer (ACRC). J Clin Oncol 23 (Suppl 16): abs. 3518, 2005.

31. Köhne CH, Van Cutsem E, Wils J, et al: Phase III study of weekly high-dose infusional fluorouracil plus folinic acid with or without irinotecan in patients with metastatic colorectal cancer: European organization for research and treatment of cancer gastrointestinal group study 40986. J Clin Oncol 23: 4856-4865, 2005. 